\title{
Interactions between Internal and External Quality Assurance in Higher Education Institutions in South Africa - A Case Analysis of Developmental Challenges Facing Academics
}

\author{
Alice Sefora Mkuzangwe \\ Tennyson Mgutshini
}

\section{Abstract}

In a world that calls for multi-skilled graduates, quality and quality assurance present serious and noteworthy challenges for higher education institutions and governments globally. Not only is quality viewed as an elusive concept with varied interpretations, but quality assurance involves multiple stakeholders and role-players whose roles in the setting and regulation of standards are varied and require in-depth understanding. The study explored the range and nature of interactions and engagements between internal institutional quality systems of the university concerned, University X, the relevant professional body, the South African Council for Social Service Professions (SACSSP) and the Council on Higher Education (CHE) with respect to internal and external quality assurance. The interactions and relationships were explored at program level through the internal and external instruments of program reviews and accreditation, using one program, the Bachelor of Social Work (BSW), as an exemplar. An exploratory qualitative design was adopted in which a combination of documents and in-depth individual interviews were used to collect data to gain a deeper understanding of both internal and external quality assurance. The content analysis method was applied on secondary data and thematic analysis was applied on primary data collected through interviews. The findings of the study revealed that national policies of the $\mathrm{CHE}$ and the policies of the institution, University $\mathrm{X}$, are in alignment, and that there is 


\section{Alice Sefora Mkuzangwe \& Tennyson Mgutshini}

misalignment between the CHE and professional bodies in general. Furthermore, the findings revealed that there was fragmentation within University $\mathrm{X}$ at policy implementation levels and that the different units responsible for quality assurance operate in silos. This study therefore recommends that quality assurance should be viewed as a system of interconnectedness between various stakeholders and role-players, in other words, an all-encompassing process to promote collaboration towards higher levels of quality in higher education.

Keywords: quality, accreditation, higher education, professional bodies, policy, legislation

\section{Introduction}

The issues of quality and quality assurance have become continous themes in higher education. Quality assurance needs to be understood within the context of the interrelatedness of many components, comparable to an ecosystem. Defined in biological terms, an ecosystem is a system that includes all living organisms in an area as well as its physical environment, and all these living organisms must co-exist and function together as a unit.

To understand quality assurance, it is useful to define the linkages and relationships between actors and role-players in QA in global, continental, national and institutional contexts. At each level various actors seek to promote outcomes that can assure standards and meet pre-determined expectations of state and society.

The challenges associated with the provision of quality education are further compounded by the perceived imposition by government at an institutional levels of accountability and comparability of programme offerings in terms of the articulation of best practice and standards in order to conform to national and even international qualification frameworks.

Development or improvement of relationships is dependent on the existence of a harmonious system that recognises all the role-players, and the delineation of roles is critical in better understanding how institutions should position themselves, as most quality assurance policies seek the participation of institutions to drive the development and implementation of policy. 
Quality assurance activities within higher education institutions should involve all key role- players, ranging from institutional leadership, academic and administrative staff, students, external authorities and society at large, and within this, the definition of quality should be informed by the institutional context shaped by the institutional quality culture. In addition, there is a need for external regulation that recognises the full spectrum of quality mechanisms which are employed in an integrated approach to quality assurance to support students as key primary role-players at each stage of their academic life cycle.

\subsection{The Centrality of Quality and Quality Assurance in the Higher Education Sector}

The centrality of quality assurance in higher education, particular, issues associated with the quality of academic programmes, standards, graduate outcomes and society's value output for their investments in higher education have become major themes.

Kayombo (2015:28) further suggests that effective quality assurance systems transcend the institutions themselves; these themes are also found in international strategies for higher education on the African continent. Harvey and Green (1993:12) locate quality as a relative concept and posit that there are two senses in which quality is relative; quality as relative to the user of the term and quality as relative to the circumstances in which it is invoked. Ansah, Swanzy and Nudzor (2017:27) identify that quality has multiple facets which demand alignment of different views, and this positions quality as a subjective phenomenon with varying opinions from internal and external stakeholders.

Conflicting relationships, or at least, tensions exist between internal and external quality assurance systems (Paintsil 2016:18), and the propensity of this relationship is to exacerbate tension and conflict rather than bring about accord and alignment between quality assurance governance structures. Green (1994) and Naidoo (2009:4) identify that quality assurance is characterised by either tensions, consensus or indifference, and that quality assurance is in a constant state of flux. The internal institutional quality assurance mechanisms and external quality assurance through government policy interact with and influence one another constantly; this relationship determines interactions within the institution and the nature of engagement with external regulatory initiatives. External quality assurance often has purposes that go beyond 


\section{Alice Sefora Mkuzangwe \& Tennyson Mgutshini}

protecting the standard of qualifications or programmes offered by universities while they strive for excellence in the production of knowledge for the public good. As quality assurance occupies not only national but continental and international spaces, the tensions in the relationships, if not adequately managed or harmonious, can have negative effects on the comparability of academic standards and national qualification frameworks which facilitate mobility of students, informational and intellectual resources across countries.

Rowlands (2012:98) argues that external quality assurance bodies often tacitly or even overtly act as agents of control, empowerment and transformation, and simultaneously as agents of the state, though not necessarily all to the same extent. A quality assurance system should recognise the need for an institution to accept responsibility for its own quality management processes, and this represents the difference between quality assurance and inspection or evaluation of quality (Sallis, 2014: 16). Quality assurance should be viewed as a total, holistic both as an internal and external process concerned with ensuring the integrity of outcomes, and places the responsibility for quality within the institution itself. By that account, it should be expressed through the institution's relationship with its internal and external stakeholders, such as students and society at large. At the same time, external quality assurance regimes should recognise the autonomy of institutions and seek to enhance institutional capacity to operate in a responsive way. It remains an institution's responsibility to assure the quality of its programme offerings and the knowledge they produce. Quality assurance activities within institutions should involve all key role- players, ranging from institutional leadership, academic and administrative staff, students, external authorities and society at large, and within this and the definition of quality should be informed by the institutional context shaped by the institutional quality culture.

In addition, there is a need for external regulation that recognises the full spectrum of quality mechanisms which are employed in an integrated approach to quality assurance to support students as key primary role-players at each stage of their academic life cycle.

There exist generic models for developing and designing institutionally based quality assurance systems that assist institutions to respond to the demands imposed by external quality regulators, governments and society. Billing (2004:114) highlights that generic models for quality assurance provide a starting point for institutions from which to map quality expectations; variations in institutional quality assurance systems are 
determined by institutional contexts and practicalities. Quality blueprints are informed by the national context of the higher education sector of that specific country. Billing (2004) further notes that these generic models are informed and shaped by the size of the higher education sector in a country, the rigidity or flexibility of the legal expression of quality assurance, or the absence of enshrinement in law, and the stage of development from state control to selfregulation of the sector.

\subsection{Quality Assurance Framework in South Africa}

Martin (2009:253) provides an analyis that the South African higher education context is mindful of past disparities in terms of education provision and further identifies that there have been systematic attempts to connect the quality question with other social purposes such as redress and access linked to issues of equity, social justice and democracy in a broader understanding of social accountability. Due to its past and its history of fragmentation, the South African higher education system presents a myriad of complex challenges in relation to quality provision of educational offerings and recognition and the purposes of quality inform the quality assurance discourse in the country.

In South Africa, the Council on Higher Education (CHE) is the statutory external body assigned to oversee quality assurance activities of the higher education sector. The CHE discharges its quality mandate through a permanent sub-committee, the Higher Education Quality Committee (HEQC). The CHE (2017) emphasises that external quality assurance offers a means of ensuring that higher education institutions have systems in place to maintain or improve the quality of their activities and educational provision. External quality assurance in the country is located at the intersection of two entities, namely the professional councils for which universities provide students equipped with unique professional knowledge, technical skills and competencies, and national quality councils mandated by government to oversee quality in institutions of higher learning. Marock (2000:43) identifies that a regulatory professional council or body is established by government to regulate qualifications and training for a particular occupation, for example, the Engineering Council of South Africa (ECSA), the Health Professions Council of South Africa (HPCSA), and, importantly for the purposes of this study, the South African Council for Social Service Professions (SACSSP). 
A strong linkage and relationship between institutions, the CHE and these various profesional councils must be establised and maintained to promote and safeguard the accreditation of programmes where such a requirement for programme accreditation has direct implications for the institution and its students in terms of programme quality, qualification recognition, professional practice registration and employability of students.

\subsection{Purpose of the Study}

The study explored the interactions between external and internal quality systems and aimed to analyse the levels of impact on each system and the relationships between role-players as informed by their own specific contexts.

The study focused on one South African university, through its internal quality instruments, how they interact with and respond to the external quality assurance requirements that are obligatory and set by external monitoring and quality assurance agencies.

The study was constructed on several assumptions, firstly, that quality assurance constitutes a steering instrument by government to drive the attainment of certain goals by institutions. The second assumption is the coexistence of relations and tensions between internal and external quality assurance measures at policy and institutional levels.

Furthermore, this study sought to explore and clarify the relations between internal and external quality assurance on how they are structured and experienced and if they are appropriately aligned to promote the assurance of positive outcomes in the accreditation processes of programmes.

The research questions that this study seeks to answer are:

i. What is the range and nature of institutional policies on quality assurance with regards to their alignment to national policy and the CHE`s Framework for Programme Accreditation?

ii. What are the interactions of role-players involved in internal and external quality assurance and how do they interact in terms of policy, procedures and implementation? 


\section{Methodology}

The concept of quality assurance can be interpreted as an uncomfortable mix of improvement, accountability, enhancement and compliance. There are contradictions in its purposes and its interpretations are informed by the relations among its actors, role-players and stakeholders. The role-players' interpretations of quality assurance are informed by their located contexts and the research questions highlight the possible contradictions and nuances between various purposes and relationships of control, improvement and accountability contextualised within a higher education institution and external QA regulation. The study adopted the interpretivism research paradigm which encompasses an interpretive stance and methodologies whose suppositions effected the ontological (how the environment is viewed) and epistemological grounding for this study. The interpretivist paradigm was chosen as the most fitting; at it has its roots in the German intellectual traditions of hermeneutics and phenomenology (Blaikie \& Priest 2019). The study undertook an investigation into whether and how, in the view of both internal institutional policies and role-players and external quality assurance bodies, the purposes of the programme accreditation processes as a quality assurance instrument have been satisfied, and included an assessment of:

i. the range and nature of institutional policies on quality assurance with regards to their alignment to national policy and the CHE Framework for Programme Accreditation and

ii. where and how the interaction of role-players involved in internal and external quality assurance take place in terms of policy, procedures and implementation;

The research approach was segmented in two interrelated phases for data collection purposes. Phase one involved document analysis to contextualise the research phenomenon in the targeted research site and this involved analysing purposively selected strategic documents, reports and national legislative documents on quality assurance, frameworks and instruments. The second phase included the use an interview protocol for individual interviews to collect data from a wide range of role-players.

The population of the study consisted of Senior and middle management staff, academic staff members, Professional and administrative 
staff who participate in the institutional committees overseeing quality assurance, approval and management structures for academic programmes from University X. The External participants population included professional body and $\mathrm{CHE}$ institutional senior managers and accreditation managers who were identified as research respondents. Quota sampling was considered as a type of purposive sampling and selection of participants and a population size of $n=16$ participants was identified. The selection and sampling of participants was largely based on expertise and knowledge of quality assurance, content knowledge of programme accreditation, academic and institutional structural arrangements in terms of seniority and designation at college level, knowledge and designations in terms of quality assurance, approval and management of programmes and participation in the regulatory structures for academic programmes both internal and external in QA.

From a population size of $n=16$, a total of $n=9$ respondents agreed to participate in the study. A notable proportion of participants (34\%) fell within the category of institutional management, while two were within the category of external quality assurance representing the $\mathrm{CHE}$ and the professional body.

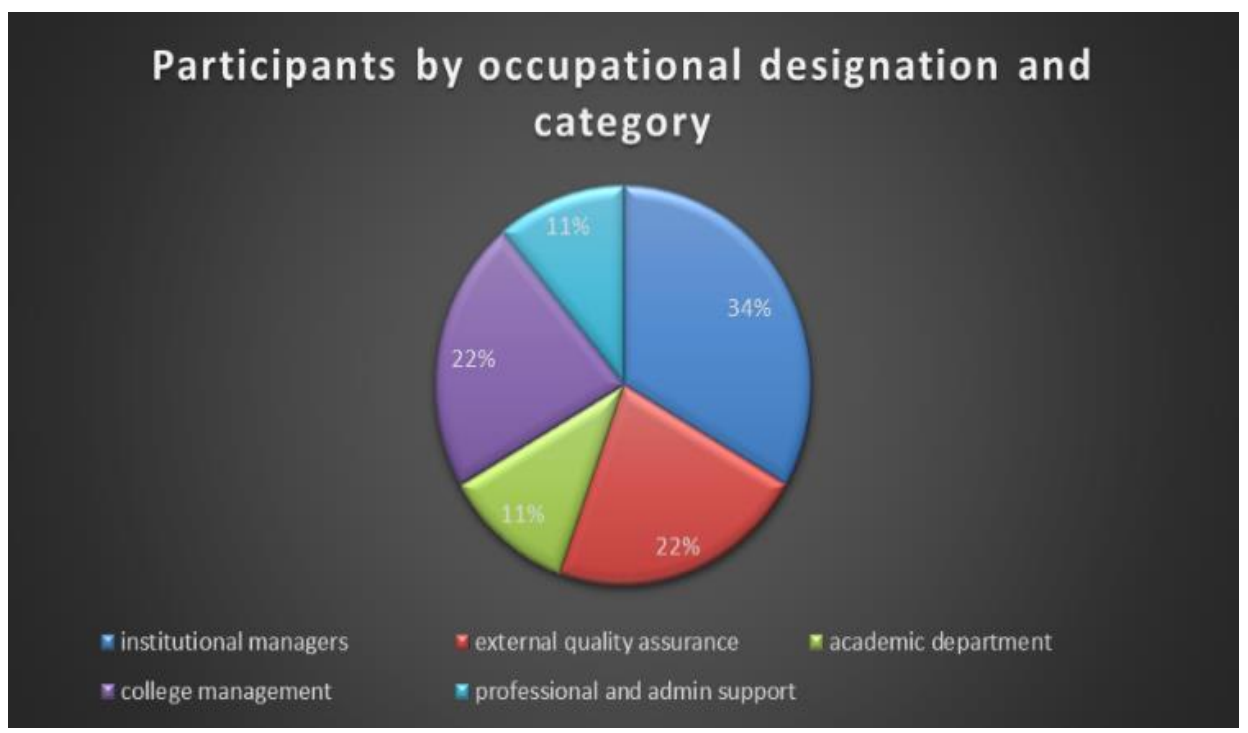

Figure 3.1: Participants by Designation and Category 
In-depth interviews were deemed optimal for collecting data on individuals' personal histories, perspectives, and experiences, particularly when sensitive topics are being explored. It is worth stating that the participants were comfortable to express their views and experiences in English. The interviewees in this study were addressed as participants in terms of presentation of their views and comments.

Data analysis combined content analysis and thematic analysis approaches for the retrospective document analysis and individual interviews $(n=9)$ respectively. The document analysis phase included a review of data elicited from policy documents and reports. Notably, simple thematic analysis was applied to analyse the feedback elicited from the study participants in the interviews. The thematic analysis involved looking beyond what was said during the interviews and specifically focused on identifying and examine the underlying ideas, perceptions, assumptions, and conceptualisations of the respondent's experiences in their current context of quality assurance.

A matrix of relational responses was developed manually to identify possible pairing of all variables; a manual approach was regarded as appropriate. Themes were identified and extracted to segment the main responses for reporting purposes. The themes were then grouped and linked to four main categories identified in terms of participant categories of Senior Management, Faculty Management, Academic Staff and Professional and Administrative Middle Management.

Once the data was paired with themes identified and segmented the researcher further interrogated if there were any emergent factors that shaped interactions of actors with the cultures and structures at individual entities, the institution, the CHE and SACSSP. This was done by examining in the datasets if the respective respondents elaborated on these factors or the environment.

\section{Findings}

The study sought to explore and examine the relations between internal institutional and external quality assurance, what the nature of the interaction is and the mechanics that drive these processes..A total of nine participants $(n=9)$ were interviewed. Interactions with such a small sample size allowed for more in-depth questions, and the qualitative nature of the engagements allowed for deeply illuminating responses and detail, i.e. answers to the 'why', 'how' 
and 'what' questions.

The main research question was how one South African university through its internal quality instruments interacts and responds to the external quality assurance requirements of the $\mathrm{CHE}$ and the professional body concerned.

A notable proportion of participants (34\%) fell within the category of institutional management, while two were within the category of external quality assurance representing the $\mathrm{CHE}$ and the professional body.

\subsection{Findings from Document Analysis}

The national Acts and in particular the Higher Education Act 101 of 1997 and Education White Paper 3, A Programme for the Transformation of Higher Education, 1997 foregrounded the exploration and analysis at policy levels together with the NQF Act 67 of 2008, the White Paper for Post-school Education and Training (RSA DHET, 2014), the Social Services Professions Act of 1978 as amended and Social Work Act, 1978 as amended. In addition, the CHE policies, frameworks, reports and institutional policies of University $\mathrm{X}$ were analysed to understand the nature and range of policies that deal with QA, mainly focusing on programmes and the accreditation aspect of programmes.

The Higher Education Act 101 of 1997, as amended, drove the analysis, read together with the NQF Act 67 of 2008, the Social Services Professions Act of 1978, as amended, and the Social Work Act, 1978 as amended. The analysis attempted to identify the roles and linkages at the highest levels of government policy of the various actors in external quality assurance such as the HEQC and the Professional Body.

i. The Higher Education Act 101 of 1997, as amended, provides for a number of role- players. In particular, the Act prescribes the roles of higher education institutions and regulatory bodies such as the $\mathrm{CHE}$ and the South African Qualifications Authority (SAQA) but does not seem to acknowledge the role of professional bodies which are a critical constituent in quality assurance.

ii. An explicit relationship and alignment exist between the institution and the CHE and the HEQC. The policies of University X identify with 
the minimum standards of the HEQC for management of quality at programme level and quality assurance as a whole.

iii. The Social Service Professions Act 110 of 1978 is consistent with the National Qualifications Act of 2008 in terms of its role, but the NQF Act goes further to note that the professional body must co-operate with the relevant quality council, in this case the HEQC, in respect of programmes and quality assurance in its occupational field. The NQF Act, however, offers little direction on the delegation of roles by the HEQC to a professional body. The incoherence of these policies most probably leads to an ambiguous relationship between the HEQC and the professional body.

iv. There is no role clarity or alignment that could be located in the National Reviews Framework of the CHE, especially with regard to how the two stakeholders, i.e. the $\mathrm{CHE}$ and the professional boards align in their drive toward the attainment of the standards in programmes from a policy perspective. In terms of standard development, the CHE Qualifications Standards Framework (CHE 2011:26) clearly identifies that it convenes a working group of academics from higher education institutions with expertise in the particular field of study, normally after consultation with a representative academic body or association. It is notable that no acknowledgement is made of the involvement of the professional body but rather an academic board or association. The National Reviews Framework (CHE 2015) specifies that the scope, qualification standard and accreditation criteria specific to a particular programme review are proposed by a reference group composed of field or discipline experts in the higher education academic community. The group is selected by the CHE from nominations received from the affected institutions.

v. Both the Programme Accreditation and National Reviews Frameworks confirm that the HEQC makes its judgements independently of other national agencies and professional bodies but takes into consideration their work where issues of quality and standards are involved. There is no clarity with respect to how this consideration of the professional 
body is exercised nor an articulation of rules of engagements with the professional bodies. Only a section in the Programme Accreditation Framework (2004:9) acknowledges that the HEQC's programme accreditation system will focus on the evaluation of new programmes. Notably, it specifies that this will be carried out by the HEQC itself, through partnerships with other statutory quality councils or professional councils in higher education on the basis of memoranda of understanding (MoUs).

The CHE's accreditation model identifies and acknowledges that certain programmes have to meet the licensing and other professional or work-based requirements of statutory councils. It also affirms that the HEQC will enter into cooperation agreements with these bodies. The agreements will be regulated by means of MoUs setting out the terms of the cooperation. The HEQC will ensure that its partnership and delegation agreements will allow it to discharge its own legal obligations for programme quality. In terms of documents sourced, only one professional body possesses a signed MOU with the CHE namely the Engineering Council of South Africa (ECSA). A desktop search on the CHE's website could not locate any other MOU signed with any other professional body.

The National Reviews Framework further makes no mention of the role or inclusion of the professional body in the reference group. This group of selected nominees makes up the national review committee (NRC) and they represent a standing sub-committee of the HEQC. In terms of the CHE the NRC comprises senior academics with expertise in programme accreditation and, depending on the particular review, expert peers from within the disciplinary area of the programme. The Framework for National Reviews (CHE 2015) is also not explicit on the role of the professional boards, while it is comparatively more explicit on the relationship with the academic institutions, in that peer reviewers of the programmes are mainly drawn from institutions themselves. In terms of this framework it is observed that a stronger relationship between the $\mathrm{CHE}$ and higher education institutions exist than between the $\mathrm{CHE}$ and professional bodies.

vi. In terms of the strategic QA reports (2019) of University X the QA department recognised that the QA environment at University $\mathrm{X}$ was 
fragmented, not well coordinated, and continued to be devolved without centralised control over where responsibility and accountability lie, as it is dispersed across various QA functions. The quality management ethos of University X, in terms of its QA policy, recognises the important need for one single point of liaison between the external and internal QA environments, which in this case is the Department of Quality Assurance and Enhancement. This Department prepared and coordinated the integrated QA Policy for University X.

\subsection{Findings from the Interviews}

Phase two of the data collection process had as theoretical underpinning Dewey's theory on experience (Dewey 1938, as cited in Hasbun \& Rudolph 2016:2), which discusses the value of experience in education and posits that experience comes from the combination of continuity and interaction. Dewey (1938:22) proposed that a person's past and present experiences impact his or her future experiences (continuity) and that there are contextual influences that affect the current experiences. Phase two attempted to solicit the views and perceptions of participants in relation to their personal experiences and their involvement with the national review of the BSW programme., Purposive sampling approaches were used to choose participants who had relevant knowledge and expertise to inform the study adequately (Heckathorn 2011:363). In order to segregate the participants into appropriate categories, the interviewees were asked about the positions they held, their backgrounds in QA and key institutional functions.

It is worth stating that the participants were comfortable to express their views and experiences in English. The interviewees in this study were addressed as participants in terms of presentation of their views and comments.

i. The interviews with all categories of staff internal to the institution revealed that within University $\mathrm{X}$, there is a culture of compliance when dealing with quality assurance matters. In all categories, including institutional management, college management, academic staff, professional and administrative staff, participants agreed that QA is being dealt with as a compliance instrument.

ii. All the categories of participants agreed that the relationships with 


\section{Alice Sefora Mkuzangwe \& Tennyson Mgutshini}

iii. professional bodies are critical and very important. The college management category firmly stated that the role of SACSSP in the academic activities of the Department of Social Work is very important, so much so that they now have an academic representative at the professional body.

iv. Professional and administrative staff viewed the relationships as critical as well but highlighted that the professional bodies did not seem to follow protocol when they engaged with the institution. There is a need for improved coordination and communication. Furthermore, there are tensions whether colleges should be responsible for liaison with professional bodies directly. Professional and administrative staff further suggested that guidelines are required for the engagement with professional bodies as accountability questions arise when things go wrong. The CHE supported the view that professional bodies have a role to play and that relations are important, in particular during the standard development process of national reviews as this needs to take into consideration the requirements of the professional bodies. The CHE participant noted that there exists a 'pulling and pushing' relationship between the $\mathrm{CHE}$ and professional bodies at standard development and setting levels and that the relationships can be quite strained in practice.

The CHE representative summed up the relationship with professional bodies by highlighting that relationships between the external authorities must be negotiated at all times as QA is a negotiated space. External authorities, both the $\mathrm{CHE}$ and professional bodies, 'need to understand the context of the institution', 'understand the psyche of an individual' and 'understand the different roles'. Both the CHE and SACSSP representatives agreed that their mutual relations can be difficult at times as some of the professional body national regulations or acts still accord them powers to accredit programmes. There also seems to be a lack of understanding from the institution on how the criteria of the professional body and of the CHE differ, and that the professional acts at policy level are not explicit and need to be reviewed.

The conceptual framing within the various roles was confirmed in the research findings as participants noted the tensions that exist between the roles of different role-players in the implementation of the various national policies, 
and that functions, processes and procedures do not translate into effective policy implementation at institutional level. These internal tensions have a direct influence on how the institution engages and relates with external agencies, in particular the professional body.

\section{Discussion}

The multi-layered nature of quality assurance allows for various role-players to interact at various levels. Harman (1998:357) notes that tensions exist between national QA and QA at institutional levels. Harman (1998:345) identifies that universities operate within a national framework and specific national educational systems, regulations and procedures adapted to meet the needs of the domestic economy and culture of their respective countries. The exploratory focus of the study established the nature of the institutional QA related polices of University $\mathrm{X}$ and the alignment of these policies to national QA policies of government, the CHE and the professional body, SACSSP.

The findings demonstrate that tensions experienced between institutional instruments and national frameworks occur due to the external location of quality and the high levels of currency afforded to external frameworks as interpreted from government policies and various external stakeholders.

The findings of the study further found that quality assurance should not be viewed as an external imposition, but rather that these national QA frameworks must be viewed by institutions as blueprints that can be used to model their own internal quality assurance instruments.

From the document analysis, the emergent main principle was that the primary responsibility for programme and institutional quality resides with higher education institutions themselves.

The findings of the study highlight that quality assurance should be viewed as a total, holistic process, concerned with ensuring the integrity of engagements at various levels of the a QA process. Quality assurance frameworks should place the responsibility for quality within the institutions themselves; this responsibility should be expressed through the relationships between internal and external stakeholders of the institutions themselves.

The findings identified that role-players involved in QA interact at policy implementation levels through procedures and processes of QA. The findings further revealed the nature of the interactions and showed that QA and 


\section{Alice Sefora Mkuzangwe \& Tennyson Mgutshini}

programme accreditation at University $\mathrm{X}$ are observed as compliance driven processes, and that institutional role-players were primarily focused on complying with the technical requirements of the process. It was further identified that at University $\mathrm{X}$ there is a strong culture of compliance when dealing with programme accreditation matters, that QA is being dealt with as a compliance instrument. The lack of acknowledgement of the quality assurance role of professional bodies in the Higher Education Act 101 of 1997, as amended, suggests that a higher premium is placed at policy level on the HEQC and the CHE. Further, an explicit relationship and alignment exist between the institution and the CHE and the HEQC.

The findings from body policy analysis and the interviews conclude that quality assurance is a system of interconnectedness guided by policies and informed by the nature of the relationships among relevant role-players. From an institutional perspective the findings further concludes that institutions do not exist in isolation, but that they must build strong relations with external authorities in the assurance of the quality of their programmes.

\section{Limitations of the study}

The study was conducted in one higher education institution in South Africa, the findings of the qualitative study may not be transferable to other situations or institutional cases as the findings are informed by context.

\section{Recommendations for Future studies}

Whilst deliberating on the findings of this study, there are several opportunities for future research to continue exploring the relationships, interaction and individual factors impeding or promoting the optimal recognition of various role players in quality assurance.

Other areas of quality assurance such institutional accreditation, programme reviews and quality promotion and the intercsecting nature of these activities can be explored since quality assurance is maultilayered. The various role players and functions of quality assurance activities in assisting institutions to be be favourably positioned to respond to external demands of quality assurance at various levels of policy and implementation, the findings of the study do allow for future qualitative exploration. 


\section{Acknowledgements}

The authors would like to aknowledge Prof. Magda Fourie-Malherbe at the Faculty of Education, Stellenbosch University for her valuable insights and support to the study.

\section{References}

Ansah, F., P. Swanzy \& N.P. Nudzor 2017. Balancing the Focus of Quality Assurance Frameworks of Higher Education Institutions in Africa: A Ghanaian Context. Global Voices in Higher Education 27. Available at: https://doi.org/10.5772/intechopen.68665

Billing, D. 2004. International Comparisons and Trends in External Quality Assurance of Higher Education: Commonality or Diversity? Higher Education 47,1: 113 - 137.

https://doi.org/10.1023/B:HIGH.0000009804.31230.5e

CHE (Council on Higher Education) 2004. Framework for Programme Accreditation. Available at:

http://www.che.ac.za/sites/default/files/publications/Programme Accredit ation Framework Nov2004.pdf (Accessed on 3 July 2017.)

CHE (Council on Higher Education) 2011. A Framework for Qualification Standards in Higher Education. Available at:

http://www.che.ac.za/sites/default/files/publications/QEP\%20Framework \%20Feb\%202015.pdf (Accessed on 9 May 2019.)

CHE (Council on Higher Education) 2015. South Africa. Framework for National Reviews. Available at:

http://www.che.ac.za/sites/default/files/publications/QEP\%20Framework \%20Feb\%202015.pdf (Accessed on 09 May 2019.)

Dewey, J. 1938. Experience and Education. Available at:

http://www.schoolofeducators.com/wp-

content/uploads/2011/12/EXPERIENCE-EDUCATION-JOHN-

DEWEY.pdf [2019

(Accessed on 4 October 2017.)

Green, D. 1994. What is Quality in Higher Education? Bristol: Taylor \& Francis.

Harman, G. 1998. The Management of Quality Assurance: A Review of International Practice. Higher Education Quarterly 52,4: 345 - 364. https://doi.org/10.1111/1468-2273.00104 
Harvey, L. \& D. Green 1993. Defining Quality. Assessment \& Evaluation in Higher Education 18,1: 9 - 34.

Hasbun, T.C. \& A. Rudolph 2016. Navigating the Waters of Accreditation: Best Practices, Challenges, and Lessons Learned from One Institution. SAGE Open 6,2: 215.

https://doi.org/10.1177/2158244016656719

Heckathorn, D.D. 2011. Comment: Snowball versus Respondent-driven Sampling. Sociological Methodology 41,1: 355 - 366.

https://doi.org/10.1111/j.1467-9531.2011.01244.x

Kayombo, J.J. 2015. Strategic Harmonization of Higher Education Systems in Africa: The Dominance of Cross-State Organizations, Government Treaties and International Conferences in Higher Education Accreditation and Quality Assurance. Journal of Literature, Languages and Linguistics 5: 26 $-30$.

Labuschagne, A. 2003. Qualitative Research-Airy-Fairy or Fundamental? The Qualitative Report 8,1: 100 - 103.

Marock, C. 2000. Quality Assurance in Higher Education: The Role and Approach of Professional Bodies and SETAs to Quality Assurance. Integrated Summary. A Report commissioned by the Higher Education Quality Committee. Unpublished.

Martin, M. 2009. On the Relationship of External Quality Assurance and Equity: Can they Converge on National Policy Agendas? Quality in Higher Education 15,3: 262.

https://doi.org/10.1080/13538320903343156

Naidoo, D. 2009. Organisational Culture and External Quality Assurance, Doctoral dissertation, Stellenbosch: University of Stellenbosch.

Paintsil, R. 2016. Balancing Internal and External Quality Assurance Dynamics in Higher Education Institutions. Master's thesis. Universitetet Oslo, Norway.

RSA (Republic of South Africa) 2010. Higher Education Act, no. 101 of 1997 as Amended. South Africa: Pretoria: Government Printers.

RSA DHET (Republic of South Africa. Department of Higher Education and Training) 2014. White Paper for Post-school Education and Training. South Africa. Available at:

http://www.dhet.gov.za/SiteAssets/Latest\%20News/White\%20paper\%20f or\%20post-school\%20education\%20and\%20training.pdf (Accessed on 05 May 2019.) 
Interactions between Internal and External Quality Assurance in HEIs

Rowlands, J. 2012. Accountability, Quality Assurance and Performativity: The Changing Role of the Academic Board. Quality in Higher Education 18,1: 97 - 110. https://doi.org/10.1080/13538322.2012.663551

Sallis, E. 2014. Total Quality Management in Education. London: Routledge. https://doi.org/10.4324/9780203417010

Alice Sefora Mkuzangwe Department of Academic Planning University of South Africa Pretoria, South Africa mkuzasa@unisa.ac.za

Tennyson Mgutshini Department of Health Studies University of South Africa Pretoria, South Africa. mgutst@unisa.ac.za 